\title{
Restricted Classes of Utility Functions for Simple Negotiation Schemes: Sufficiency, Necessity, and Maximality
}

\author{
Yann Chevaleyre*, Ulle Endriss ${ }^{\dagger}$, Nicolas Maudet*
}

\begin{abstract}
Résumé
Dans ce papier, nous étudions les propriétés d'un cadre abstrait de négociation dans lequel les agents négocient à propos de l'allocation de ressources discrètes. Dans la mesure où l'atteinte d'une allocation optimale peut nécessiter l'utilisation de transactions extrêmement complexes, on s'intéresse à l'identification de classes de fonctions d'utilité permettant de garantir une issue optimale à la négociation, même si celle-ci est conduite à l'aide uniquement de transactions simples impliquant une seule ressource à chaque fois. On montre que la classe des fonctions modulaires n'est pas seulement suffisante (lorsque les paiements compensatoires sont permis), mais aussi maximale. Un résultat similaire est proposé dans le cadre des négociations sans paiements compensatoires.
\end{abstract}

Mots-clefs : Allocation de Ressources Distribuée, Négociation Automatique

\begin{abstract}
We investigate the properties of an abstract negotiation framework where agents autonomously negotiate over allocations of discrete resources. In this framework, reaching an optimal allocation potentially requires very complex multilateral deals. Therefore, we are interested in identifying classes of utility functions such that any negotiation conducted by means of deals involving only a single resource at at time is bound to converge to an optimal allocation whenever all agents model their preferences using these functions. We show that the class of modular utility functions is not only sufficient (when side-payments are allowed) but also maximal in this sense. A similar result is proven in the context of negotiation without money.
\end{abstract}

Key words : Multiagent Resource Allocation, Negotiation

*LAMSADE, Université Paris-Dauphine, 75775 Paris cedex 16. \{chevaley, maudet \}

@ lamsade.dauphine.fr

${ }^{\dagger}$ Institute for Logic, Language and Computation, University of Amsterdam. ue@doc . ic . ac. uk 


\section{Introduction}

The problem of discrete resource allocation has recently received much attention from the artificial intelligence community. A large amount of this work is focused on combinatorial auctions [5]. In this case, the allocation procedure is centralised, and the so-called winner determination problem consists in determining the allocation of resources maximising the auctioneer's revenue.

A different perspective is taken when one assumes that the allocation process is truly distributed, in the sense that agents autonomously negotiate over the bundles of resources they hold. This assumption is justified in many applications where no central authority can be relied on to decide upon the allocation of resources. In this case, the system designer will typically seek to set up the system in such way that it guarantees desirable properties, without directly interfering in the negotiation process itself. In this paper we will make use of such an abstract negotiation framework investigated by a number of authors $[12,7,6]$.

To make things more precise, we assume a set of negotiating agents populating the system, and we model their preferences (over different bundles of resources) by means of utility functions. In order to pursue their own interests, agents agree on deals benefitting themselves but without planning ahead (i.e. they are both rational and myopic), thereby modifying the allocation of resources. From a global point of view, the quality of an allocation reflects the overall performance of the system, and the designer will naturally seek to ensure that negotiation converges towards an optimal allocation.

Section 2 introduces the negotiation framework used in this paper. As we shall recall in Section 3, it is known that very complex multilateral deals are potentially required to reach an optimal allocation. When deals are restricted (e.g. to a limited number of resources), it is only possible to guarantee an optimal outcome by also restricting the negotiation process to agents whose preferences have certain properties. In this paper, we study the conditions under which negotiation conducted by means of the simplest deals, involving one item at a time (or 1-deal negotiation for short) still allows us to reach an optimal allocation. Section 5 generalises a result from the literature and shows that modelling preferences with modular utility functions is a sufficient condition. However, modularity is not a necessary condition. This is demonstrated in Section 6.2 by means of a counterexample. We also show that there can be no condition on utility functions that would be both necessary and sufficient for optimal allocations to be negotiable by means of rational 1-deals. The main contribution of this paper is to show that the class of modular utility functions is maximal, in the sense that no class strictly including the modular utility functions would still be sufficient for 1-deal negotiation. The proof detailed in Section 7 shows that, given any non-modular utility function, it is always possible to construct a modular utility function and select a scenario where the optimal allocation cannot be reached by 1 -deals. Section 8 concludes. 


\section{Myopic Negotiation Over Resources}

In this section, we introduce the decentralised negotiation framework used throughout this paper and report a number of known technical results. In this framework, a finite set of agents negotiate over a finite set of discrete (i.e. non-divisible) resources. A resource allocation is a partitioning of the resources amongst the agents (that is, every resource has to be allocated to one and only one agent). As an example, the allocation $A$ defined by $A(i)=\left\{r_{1}\right\}$ and $A(j)=\left\{r_{2}, r_{3}\right\}$ would allocate resource $r_{1}$ to agent $i$, while resources $r_{2}$ and $r_{3}$ would be owned by agent $j$.

We are going to model the preferences of agents by means of utility functions mapping bundles of resources to real numbers. Assuming that agents are only concerned with resources they personally own, we will use the abbreviation $u_{i}(A)$ for $u_{i}(A(i))$, representing the utility value assigned by agent $i$ to the bundle it holds for allocation $A$. The parameters of a negotiation problem are summarised in the following definition:

Definition 1 (Negotiation problems) A negotiation problem is a tuple $\mathcal{P}=\left\langle\mathcal{R}, \mathcal{A}, \mathcal{U}, A_{0}\right\rangle$, where

- $\mathcal{R}$ is a finite set of indivisible resources;

- $\mathcal{A}=\{1, \ldots, n\}$ is a finite set of agents $(n \geq 2)$;

- $\mathcal{U}=\left\langle u_{1}, \ldots, u_{n}\right\rangle$ is a vector of utility functions, such that for all $i \in \mathcal{A}, u_{i}$ is a mapping from $2^{\mathcal{R}}$ to $\mathbb{R}$;

- $A_{0}: \mathcal{A} \rightarrow 2^{\mathcal{R}}$ is an (initial) allocation.

Agents may agree on a deal to exchange some of the resources they possess. It transforms the current allocation of resources $A$ into a new allocation $A^{\prime}$; that is, we can define a deal as a pair $\delta=\left(A, A^{\prime}\right)$ of allocations (with $A \neq A^{\prime}$ ).

We should stress that this is a multilateral negotiation framework. A single deal may involve the displacement of any number of resources between any number of agents. An actual implementation of this abstract framework may, however, not allow for the same level of generality. Sandholm [12] has proposed a typology of different types of deals, such as swap deals involving an exchange of single resources between two agents or cluster deals involving the transfer of a set of items from one agent to another. The simplest type of deals are those involving only a single resource (and thereby only two agents).

Definition 2 (1-deals) $A$ 1-deal is a deal $\delta=\left(A, A^{\prime}\right)$ resulting in the reallocation of exactly one resource. 
The above is a condition on the structure of a deal. Other conditions relate to the $a c$ ceptability of a deal to a given agent. We assume that agents are rational in the sense of aiming to maximise their individual welfare. Furthermore, agents are assumed to be myopic. This means that agents will not accept deals that would reduce their level of welfare, not even temporarily, because they are either not sufficiently able to plan ahead or not willing to take the associated risk (see also [12] for a justification of such an agent model in the context of multiagent resource allocation).

\subsection{Negotiating With Money}

In the first variant of this framework, we will permit agents to enhance deals with monetary side payments, in order to compensate other agents for a possible loss in utility. This can be modelled using a payment function $p: \mathcal{A} \rightarrow \mathbb{R}$. Such a function has to satisfy the side constraint $\sum_{i \in \mathcal{A}} p(i)=0$, i.e. the overall amount of money in the system remains constant. If $p(i)>0$, then agent $i$ pays the amount of $p(i)$, while $p(i)<0$ means that it receives the amount of $-p(i)$. The following rationality criterion will define the acceptability of deals:

Definition 3 (Individual rationality) $A$ deal $\delta=\left(A, A^{\prime}\right)$ is rational iff there exists a payment function $p$ such that $u_{i}\left(A^{\prime}\right)-u_{i}(A)>p(i)$ for all $i \in \mathcal{A}$, except possibly $p(i)=0$ for agents $i$ with $A(i)=A^{\prime}(i)$.

From a system designer's perspective, we are interested in assessing the well-being of the whole society, or social welfare [1], which is often defined as the sum of utilities of all the agents.

Definition 4 (Social welfare) The social welfare $s w(A)$ of an allocation $A$ is defined as follows:

$$
s w(A)=\sum_{i \in \mathcal{A}} u_{i}(A)
$$

This is the utilitarian definition of social welfare. While this is the definition usually adopted in the multiagent systems literature [14], we should stress that also several of the other notions of social welfare developed in the social sciences (e.g. egalitarian social welfare [1]) do have potential applications in the context of multiagent resource allocation.

We conclude this background section by recalling two important results $[12,7]$ : the first one makes explicit the connection between the local decisions of agents and the global behaviour of the system, and the second one is the fundamental convergence theorem for this negotiation framework. 
Lemma 1 (Individual rationality and social welfare) $A$ deal $\delta=\left(A, A^{\prime}\right)$ is rational iff $s w(A)<s w\left(A^{\prime}\right)$.

Theorem 1 (Maximising social welfare) Any sequence of rational deals will eventually result in an allocation of resources with maximal social welfare.

The main significance of this latter result, beyond the equivalence of rational deals and social welfare-increasing deals stated in Lemma 1, is that any sequence of deals satisfying the rationality criterion will eventually converge to an optimal allocation. There is no need for agents to consider anything but their individual interests. Every single deal is bound to increase social welfare and there are no local minima.

\subsection{Negotiating Without Money}

What happens if we do not allow agents to enhance deals with monetary side payments (or explicit utility transfers), in order to compensate other agents for a possible loss in utility? In this context, we shall assume that agents are cooperatively rational in the sense of accepting deals that may not result in a strict increase in personal welfare, with the further condition that at least one agent will strictly benefit from the deal.

Definition 5 (Cooperative rationality) $A$ deal $\delta=\left(A, A^{\prime}\right)$ is called cooperatively rational iff $u_{i}(A) \leq u_{i}\left(A^{\prime}\right)$ for all $i \in \mathcal{A}$ and there exists an agent $j \in \mathcal{A}$ such that $u_{j}(A)<u_{j}\left(A^{\prime}\right)$.

Note that we have $s w(A)<s w\left(A^{\prime}\right)$ for any deal $\delta=\left(A, A^{\prime}\right)$ that is cooperatively rational, but not vice versa. Clearly, in this general setting, it is not possible to guarantee that agents will eventually reach a socially optimal allocation of resources. It is not hard to show, though, that a Pareto optimal allocation can always be reached (recall that the number of allocations is finite). This holds when any type of deals are allowed. Only the slightest restriction on the number of agents and/or resources allowed to be involved in a deal would prevent this result [7].

To compensate for this, one possible solution is to also restrict the class of utility functions at hand when representing the preferences of agents. More precisely, we will investigate in this paper the sufficiency of a given class of utility functions when only the simplest deals are allowed, in the sense that for any set of utility functions $\left\{u_{1}, \ldots, u_{n}\right\}$ drawn from this class, any sequence of rational one-resource-at-a-time deals will eventually result in an allocation of resources with maximal social welfare. For the sake of brevity, we say from now on that such a class permits 1-deals. We will then ask the question of the necessity of this class of utility functions, and give a general argument that will 
motivate us to investigate instead the maximality property of this class. The classes of utility functions we will be interested in are the classes of modular functions with shared $\alpha, \beta$-values functions, or $\mathcal{M}_{\alpha, \beta}$ functions for short.

\section{Reachability For Restricted Classes of Utility Functions}

While Theorem 1 shows that, in principle, it is always possible to negotiate an allocation of resources that is optimal from a social point of view, deals involving any number of agents and resources may be required to do so $[12,7]$. In particular, the most basic type of deal, which involves moving a single resource from one agent to another and which is the type of deal implemented in most systems realising a kind of Contract Net protocol [13], is certainly not sufficient for negotiation between agents that are not only rational but also myopic.

This has first been shown by Sandholm [12] and is best explained by means of an example. ${ }^{2}$ Let $\mathcal{A}=\{1,2,3\}$ and $\mathcal{R}=\left\{r_{1}, r_{2}, r_{3}\right\}$. Suppose the utility functions of these agents are defined as follows (over singleton sets):

$$
\begin{array}{lll}
u_{1}\left(\left\{r_{1}\right\}\right)=5 & u_{1}\left(\left\{r_{2}\right\}\right)=1 & u_{1}\left(\left\{r_{3}\right\}\right)=0 \\
u_{2}\left(\left\{r_{1}\right\}\right)=0 & u_{2}\left(\left\{r_{2}\right\}\right)=5 & u_{2}\left(\left\{r_{3}\right\}\right)=1 \\
u_{3}\left(\left\{r_{1}\right\}\right)=1 & u_{3}\left(\left\{r_{2}\right\}\right)=0 & u_{3}\left(\left\{r_{3}\right\}\right)=5
\end{array}
$$

Furthermore, for any bundle $R$ not listed above, suppose $u_{i}(R)=0$ for all $i \in \mathcal{A}$. Let $A_{0}$ with $A_{0}(1)=\left\{r_{2}\right\}, A_{0}(2)=\left\{r_{3}\right\}$ and $A_{0}(3)=\left\{r_{1}\right\}$ be the initial allocation, i.e. $s w\left(A_{0}\right)=3$. The optimal allocation would be $A^{*}$ with $A^{*}(1)=\left\{r_{1}\right\}, A^{*}(2)=\left\{r_{2}\right\}$ and $A^{*}(3)=\left\{r_{3}\right\}$, which yields a social welfare of 15 . All other allocations have lower social welfare than $A^{*}$. Hence, starting from $A_{0}$, the deal $\delta=\left(A_{0}, A^{*}\right)$ would be the only deal increasing social welfare. By Lemma $1, \delta$ would also be the only rational deal. This deal, however, involves all three resources and affects all three agents. In particular, $\delta$ is not a 1-deal. Hence, if we choose to restrict ourselves to rational deals, then 1-deals are not sufficient to negotiate allocations of resources with maximal social welfare.

Of course, for some particular negotiation problems, rational 1-deals will be sufficient. The difficulty lies in recognising the problems where this is so. Closely related to this issue, Dunne et al. [6] have shown that, given two allocations $A$ and $A^{\prime}$ with $\operatorname{sw}(A)<$ $s w\left(A^{\prime}\right)$, the problem of checking whether it is possible to reach $A^{\prime}$ from $A$ by means of a sequence of rational 1-deals is NP-hard in the number of resources in the system.

The structural complexity of deals required to be able to guarantee socially optimal outcomes partly stems from the generality of the framework. In particular, so far we have

\footnotetext{
${ }^{2} \mathrm{~A}$ methodology for constructing such examples is easily generated from the proof of the result on the insufficiency of any kind of structurally limited class of deals given by Endriss et al. [7].
} 
made no assumptions on the structure of utility functions used by the agents to model their preferences. By introducing restrictions on the class of admissible utility functions, it may be possible to ensure convergence to an allocation with maximal social welfare by means of simpler deals. In this paper, we are interested in characterising more precisely those classes of utility functions that permit 1-deal negotiation.

Definition 6 (1-deal negotiation from a class $\mathcal{C}$ ) Given a class $\mathcal{C}$ of utility functions and a number $n$ of agents, $\mathcal{C}$ is said to permit 1-deal negotiation iff any sequence of rational 1-deals will eventually result in an allocation of resources with maximal social welfare whenever all utility functions $\left\{u_{1}, \ldots, u_{n}\right\}$ are drawn from $\mathcal{C}$.

Under this perspective, a relevant result is due to Endriss et al. [7], who show that rational 1-deals are sufficient to guarantee outcomes with maximal social welfare in case all agents use additive utility functions. ${ }^{3}$ We are going to prove a slight generalisation of this result in the next section.

\section{Modular Utility Functions and Variants}

We are now going to define the class of modular utility functions. This is an important (see e.g. [11]), albeit simple, class of functions that can be used in negotiation domains where there are no synergies (neither complementaries nor substitutables) between different resources.

Definition 7 (Modular utility) A utility function $u$ is modular iff the following holds for all bundles $R_{1}, R_{2} \subseteq \mathcal{R}$ :

$$
u\left(R_{1} \cup R_{2}\right)=u\left(R_{1}\right)+u\left(R_{2}\right)-u\left(R_{1} \cap R_{2}\right)
$$

The class of modular functions includes the aforementioned additive functions. This may be seen as follows. Let $R$ be any non-empty bundle of resources and let $r \in R$. Then equation (1) implies $u(R)=u(R \backslash\{r\})+[u(\{r\})-u(\{\})]$. If we apply this step recursively for every resource in $R$, then we end up with the following equation:

$$
u(R)=u(\{\})+\sum_{r \in R}[u(\{r\})-u(\{\})]
$$

That is, in case $u(\{\})=0$, the utility assigned to a set will be the sum of utilities assigned to its members (i.e. $u$ will be additive). Clearly, equation (2) also implies equation (1), i.e. the two characterisations of the class of modular utility functions are equivalent.

\footnotetext{
${ }^{3} \mathrm{~A}$ utility function is additive iff the utility assigned to a set of resources is always the sum of utilities assigned to its members.
} 
We now introduce a restriction on the class of modular utility functions, namely the classes of modular functions with shared $\alpha, \beta$-values (or $\mathcal{M}_{\alpha, \beta}$ for short). Intuitively, $\mathcal{M}_{\alpha, \beta}$ classes are suited in domains where agents can only like, dislike, or possibly be indifferent with regards to resources of the system. The key point is that agents all agree on the values they may assign to each single resource. $\mathcal{M}_{\alpha, \beta}$ functions hence define a set of classes, each class being parametrized by the vectors of possible values assigned to each resource. An example of $\mathcal{M}_{\alpha, \beta}$ class would be $\mathcal{M}_{-1,+1}$ where agents simply assign -1 to each resource they dislike, +1 to each resource they like, and 0 when they are indifferent as to whether holding the resource or not.

Definition 8 (modular functions with shared $\alpha, \beta$-values) A set of utility functions $u_{1} \ldots u_{n}$ is modular with shared $\alpha, \beta$-values iff

- there exists a list of coefficients $\alpha=\left(\alpha_{r_{1}}, \ldots, \alpha_{r_{|\mathcal{R}|}}\right) \in \mathbb{R}^{|| \mathcal{R} \mid}$,

- there exists a list of coefficients $\beta=\left(\beta_{r_{1}}, \ldots, \beta_{r_{|\mathcal{R}|}}\right) \in \mathbb{R}^{-|\mathcal{R}|}$, and

- for each utility function $u_{i}$ there exist two sets $S_{i}^{+}, S_{i}^{-} \subseteq \mathcal{R}$ with $S_{i}^{+} \cap S_{i}^{-}=\{\}$, such that $\forall R \subseteq \mathcal{R}, u_{i}(R)=u_{i}(\{\})+\sum_{r \in R \cap S_{i}^{+}} \alpha_{r}+\sum_{r \in R \cap S_{i}^{-}} \beta_{r}$

By extension, given two lists of coefficients $\alpha \in \mathbb{R}^{+|\mathcal{R}|}, \beta \in \mathbb{R}^{-|\mathcal{R}|}$, the class $\mathcal{M}_{\alpha, \beta}$ is defined as the biggest set of modular functions with shared $\alpha, \beta$-values.

Consider for instance the class $\mathcal{M}_{(2,1),(-20,-7)}$ over resources $r_{1}, r_{2}$, and let $u_{1}$ and $u_{2}$ be two utility functions drawn from this class such that $u_{1}(\{\})=u_{2}(\{\})=0, S_{1}^{+}=$ $\left\{r_{1}\right\}, S_{1}^{-}=\left\{r_{2}\right\}, S_{2}^{+}=\left\{r_{2}\right\}$, and $S_{2}^{-}=\{\}$. Then, the values of $u_{1}$ and $u_{2}$ are as shown in the following table:

\begin{tabular}{|c||c|c|}
\hline$R$ & $u_{1}(R)$ & $u_{2}(R)$ \\
\hline \hline\{\} & 0 & 0 \\
\hline$\left\{r_{1}\right\}$ & 2 & 0 \\
\hline$\left\{r_{2}\right\}$ & -20 & 1 \\
\hline$\left\{r_{1}, r_{2}\right\}$ & -18 & 1 \\
\hline
\end{tabular}

\section{Sufficient Classes of Utility Functions}

\subsection{Framework With Money}

It turns out that in domains where all utility functions are modular, it is always possible to reach a socially optimal allocation by means of a sequence of rational deals involving only a single resource each. This is a slight generalisation of a result proved by Endriss $e t$ al. [7], and our proof closely follows theirs. 
Theorem 2 (Negotiation in modular domains) The class $\mathcal{M}$ of modular utility functions permits 1-deal negotiation.

Proof. By Lemma 1, any rational deal results in a strict increase in social welfare. Together with the fact that the number of distinct allocations is finite, this ensures that there can be no infinite sequence of rational deals (termination). It therefore suffices to show that for any allocation that does not have maximal social welfare there still exists a rational 1-deal that would be applicable.

We are going to use the alternative characterisation of modular utility functions given by equation (2). For any allocation $A$, let $f_{A}$ be the function mapping each resource $r$ to the agent $i$ that holds $r$ in situation $A$. Then, for modular domains, the formula for social welfare (see Definition 4) can be rewritten as follows:

$$
s w(A)=\sum_{i \in \mathcal{A}} u_{i}(\{\})+\sum_{r \in \mathcal{R}} u_{f_{A}(r)}^{\prime}(\{r\})
$$

with $u_{i}^{\prime}(R)=u_{i}(R)-u_{i}(\{\})$. Now assume we have reached an allocation of resources $A$ that does not have maximal social welfare, i.e. there exists another allocation $A^{\prime}$ with $s w(A)<s w\left(A^{\prime}\right)$. Considering the above definition of social welfare and observing that $\sum_{i \in \mathcal{A}} u_{i}(\{\})$ is a constant that is independent of the current allocation, this implies that at least one resource $r$ must satisfy the inequation $u_{f_{A}(r)}^{\prime}(\{r\})<u_{f_{A^{\prime}}(r)}^{\prime}(\{r\})$, i.e. the agent owning $r$ in allocation $A$ values that resource less than the agent owning it in allocation $A^{\prime}$. But then the 1-deal consisting of passing $r$ from agent $f_{A}(r)$ to agent $f_{A^{\prime}(r)}$ would already increase social welfare and thereby be rational.

Like Theorem 1, the above establishes an important convergence result towards a global optimum by means of decentralised negotiation between self-interested agents. In addition, provided all utility functions are modular, convergence can be guaranteed by means of a much simpler negotiation protocol, which only needs to cater for agreements on 1-deals (rather than multilateral deals over sets of resources).

\subsection{Framework Without Money}

We are now going to prove a similar result in the variant of our framework that does not allow for side-payments.

Theorem 3 (Sufficiency) Given two vectors $\alpha \in \mathbb{R}^{+|\mathcal{R}|}, \beta \in \mathbb{R}^{-|\mathcal{R}|}$, the class $\mathcal{M}_{\alpha, \beta}$ of modular functions with shared $\alpha, \beta$-values permits 1-deals.

Proof. Any rational deal results in a strict increase in social welfare. Together with the fact that the number of distinct allocations is finite, this ensures that there can be no 
infinite sequence of rational deals (termination). It therefore suffices to show that for any allocation that does not have maximal social welfare there still exists a cooperative rational one-resource-at-a-time deal that would be applicable. If an allocation $A$ does not have maximal social welfare then it must be the case that some agent $i$ holds a resource $r$ and that there is another agent $j$ in the system such that $u_{i}(\{r\})<u_{j}(\{r\}$. That is, either (i) some agent $i$ holds a resource $r$ with $u_{i}(\{r\})-u_{i}(\{\})=0$, and there is another agent $j$ in the system with $u_{j}(\{r\})-u_{i}(\{\})=\alpha_{r}$, or (ii) some agent $i$ holds a resource $r$ with $u_{i}(\{r\})-u_{i}(\{\})=\beta_{r}$, and there is another agent $j$ in the system such that either (iia) $u_{j}(\{r\})-u_{j}(\{\})=0$, or (iib) $u_{j}(\{r\})-u_{j}(\{\})=\alpha_{r}>u_{i}(\{r\})$. In every case, passing $r$ from $i$ to $j$ would be a cooperatively rational deal, so either negotiation has not yet terminated or we are in situation with maximal social welfare.

\section{Necessity Issues}

\subsection{Modularity Is Not Necessary}

In the previous section we have introduced a class of utility functions (namely modular functions) such that it is possible to guarantee that sequences of rational 1-deals with sidepayments will converge to an allocation with maximal social welfare under the condition that all agents' utilities belong to this class. A similar result has been proven in the framework without money, when all agents' utility functions belong to a proper subclass of modular functions (modular functions with shared $\alpha, \beta$-values). A natural question to ask would then be whether belonging to these classes is also a necessary condition to guarantee convergence in the respective frameworks.

It turns out that this is not the case, in both cases. We demonstrate this by means of the following example. Suppose $\mathcal{R}=\left\{r_{1}, r_{2}\right\}$ and there are two agents with utility functions $u_{1}$ and $u_{2}$ :

$$
\begin{aligned}
& u_{1}(\{\})=\begin{array}{lll}
0 & u_{2}(\{\})=0
\end{array} \\
& u_{1}\left(\left\{r_{1}\right\}\right)=10 \quad u_{2}\left(\left\{r_{1}\right\}\right)=10 \\
& u_{1}\left(\left\{r_{2}\right\}\right)=10 \quad u_{2}\left(\left\{r_{2}\right\}\right)=10 \\
& u_{1}\left(\left\{r_{1}, r_{2}\right\}\right)=0 \quad u_{2}\left(\left\{r_{1}, r_{2}\right\}\right)=0
\end{aligned}
$$

The situation is as follows: each agent is willing to hold a single resource, and has no preference as to which resource it actually holds. None of these functions is modular. The optimal allocations are the allocation where each agent holds one resource. Furthermore, as may easily be checked, any 1-deal that involves moving a single resource from agent 2 to agent 1 is rational. Hence, rational 1-deals are sufficient to move to the optimal allocation for this scenario, despite the functions not being modular. 


\subsection{There Is No Sufficient And Necessary Class}

In fact, it is possible to show that there can be no class of utility functions that would be both sufficient and necessary in this sense. It suffices to produce two concrete utility functions $u_{1}$ and $u_{2}$ such that $(i)$ both of them would guarantee convergence if all agents were using them, and $(i i)$ there is a scenario where some agents are using $u_{1}$ and others $u_{2}$ and convergence is not guaranteed. This is so, because assuming that a necessary and sufficient class exists, $(i)$ would imply that both $u_{1}$ and $u_{2}$ belong to that class, while (ii) would entail the contrary. We give two such functions for the case of two agents and two resources (the argument is easily augmented to the general case):

$$
\begin{array}{llll}
u_{1}(\{\}) & =0 & u_{2}(\{\}) & =0 \\
u_{1}\left(\left\{r_{1}\right\}\right) & =1 & u_{2}\left(\left\{r_{1}\right\}\right) & =5 \\
u_{1}\left(\left\{r_{2}\right\}\right) & =2 & u_{2}\left(\left\{r_{2}\right\}\right) & =5 \\
u_{1}\left(\left\{r_{1}, r_{2}\right\}\right)=3 & u_{2}\left(\left\{r_{1}, r_{2}\right\}\right)=5
\end{array}
$$

The function $u_{1}$ is modular, i.e. all agents using that function is a sufficient condition for guaranteed convergence to an optimal allocation by means of rational 1-deals (Theorem 1). Clearly, convergence is also guaranteed if all agents are using $u_{2}$. However, if the first agent uses $u_{1}$ and the second $u_{2}$, then the allocation $A$ with $A(1)=\left\{r_{1}\right\}$ and $A(2)=\left\{r_{2}\right\}$ is not socially optimal and the only deal increasing social welfare (and thereby, the only rational deal) would be to swap the two resources simultaneously. Hence, no condition on all agents' utility functions can be both sufficient and necessary to guarantee convergence to an optimal allocation by means of rational 1-deals alone.

\subsection{Checking a Necessary and Sufficient Condition on Sets of Utility Functions is Intractable}

In the previous section, we showed there are no class of utility functions that would be both sufficient and necessary to permit 1-deal negotiation. A natural question therefore arises : is there a simple necessary and sufficient condition on sets of utility functions instead of classes of utility functions, which permits 1-deal negotiation? Such a condition can be expressed as a decision problem over the set of utility functions, as follows:

Definition 9 (1-deal negotiation from a set $\left\{u_{1}, \ldots, u_{n}\right\}$ ) Given a set $\left\{u_{1}, \ldots, u_{n}\right\}$ of utility functions, this set is said to be permit 1-deal negotiation iff with $n$ agents sharing these utility functions, any sequence of rational 1-deals will eventually result in an allocation of resources with maximal social welfare.

We will show here that under a few restrictions, the above decision problem is co-NPhard. This means that a necessary and sufficient condition on sets of utilities would be 
intractable to check. In other words, such a condition would not be as simple as those used in previous section (e.g. modularity).

First note that such a hardness result necessarily depends on the representation language used for the utility functions. Recently, Dunne showed that for utility functions represented with SLP (Straight Line Programs), checking if sets of utilities permit 1-deal negotiation was co-NP-hard. Here, instead of showing a similar result for another particular representation, we will define some general restrictions on representation languages under which the hardness results hold.

First of all, let us define two general restrictions on representation languages, the first of which is related to the hardness of solving a decision problem (called utility improvement) defined as follows, and the second of which is concerned with the structure of the languages (called $r$-composition).

Definition 10 (Utility Improvement) Given a utility $u$ represented with a representation language $\mathcal{R E} \mathcal{P}^{\prime}$, and given a set $Q \subseteq \mathcal{R}$, the Utility Improvement (UI) problem is defined as follows: check wether there exists $Q^{\prime} \subseteq \mathcal{R}$ such that $u\left(Q^{\prime}\right)>u(Q)$.

Clearly, the hardness of the UI problem is related to that of finding the maximum of the function $u$. Of course, the utility improvement decision problem is known to be NP-hard for many representation languages, as detailed further.

Definition 11 ( $r$-composition) Let $\mathcal{R E \mathcal { P }}$ and $\mathcal{R} \mathcal{E} \mathcal{P}^{\prime}$ be two representation languages over utility functions. Let $r \in \mathcal{R}$ be a resource. Then, $\mathcal{R E \mathcal { P }}$ is said to be a $r$-composition over $\mathcal{R} \mathcal{E} \mathcal{P}^{\prime}$ iff $\forall u \in \mathcal{R E} \mathcal{P}^{\prime}, \forall k \in \mathbb{R}$, the utilities $v$ and $\bar{v}$ defined below belong to $\mathcal{R} \mathcal{E} \mathcal{P}$ and the number of bits needed to represent both utilities is polynomially bounded by the size of $u$ :

$$
v(R)=\left\{\begin{array}{cl}
u(R)-k & \text { if } r \in R \\
0 & \text { otherwise }
\end{array} \quad \bar{v}(R)=\left\{\begin{array}{cl}
u(R)-k & \text { if } r \notin R \\
0 & \text { otherwise }
\end{array}\right.\right.
$$

For the sake of simplicity, we will say that a representation $\mathcal{R E} \mathcal{P}$ is $r$-composed iff there exists a representation $\mathcal{R E} \mathcal{P}^{\prime}$ over which $\mathcal{R E} \mathcal{P}$ is a $r$-composition.

As many interesting representation languages are $r$-composed, the results presented here are quite general. Let us briefly present some of these representation languages commonly used in multiagent resource allocation. In the following, $r$ is any resource chosen among $\mathcal{R}$.

- The $k$-additive representation [9] for $k \geq 3$ is a $r$-composition over the $(k-1)$ additive representation. Intuitively, $k$-additive utility functions can be represented by a multinomial of degree $k$ [2]. Note that for $k \geq 2$, the UI problem with $k$ additive functions is NP-hard [4]. 
- Posiforms of degree $k$ [2] for $k \geq 3$ are $r$-compositions over posiforms of degree $k-1$. This is a generalization of $k$-additive forms, and thus for $k \geq 2$, the UI problem here is also NP-hard [4].

- The bundle form representation [4] is also a $r$-composition over itself. It consists in enumerating all bundles of resources for which the utility is not null. Again, it was shown in [4] that the associated UI problem is NP-hard.

- The SLP (Straight Line Programs) representation [6] is a $r$-composition over itself. Intuitively, SLP are computer programs without loops. Dunne showed that UI is also NP-hard using this representation [6].

We can now present the main theorem of this section, lying on the above restrictions.

Theorem 4 Let $\mathcal{R E} \mathcal{P}$ be a representation language. If the following conditions hold, then the problem of checking if a set of utilities $\left\{u_{1}, \ldots, u_{n}\right\}$ represented with $\mathcal{R} \mathcal{E} \mathcal{P}$ permits 1-deal negotiation is co-NP-hard, even with only two agents:

- there exists a representation $\mathcal{R} \mathcal{E} \mathcal{P}^{\prime}$ over which $\mathcal{R E \mathcal { P }}$ is a r-composition

- the UI problem with this representation $\mathcal{R} \mathcal{E} \mathcal{P}^{\prime}$ is $N P$-hard

- There exists a null valued utility function $v_{0}$ in $\mathcal{R E} \mathcal{P}$

Proof. Let $\langle u, Q\rangle$ be an instance of the UI problem over the $\mathcal{R} \mathcal{E} \mathcal{P}^{\prime}$ representation. Let us show that this problem reduces to checking if sets of utilities permit 1-deal negotiation.

Let $v_{0}$ be the null-valued utility function, let the set of agents be $\mathcal{A}=\{1,2\}$. Let us introduce two utility functions:

$$
v(R)=\left\{\begin{array}{cl}
u(R)-u(Q) & \text { if } r_{1} \in R \\
0 & \text { otherwise }
\end{array} \quad \bar{v}(R)=\left\{\begin{array}{cl}
u(R)-u(Q) & \text { if } r_{1} \notin R \\
0 & \text { otherwise }
\end{array}\right.\right.
$$

Clearly, these two functions belong to $\mathcal{R E} \mathcal{P}$, because of the $r$-composition property. We can now define two negotiation problems. Let $\mathcal{P}$ (resp. $\overline{\mathcal{P}}$ ) be the problem of checking if 1-deal negotiation is permitted, for two agents with utilities $v$ and $v_{0}$ (resp. $\bar{v}$ and $v_{0}$ ). We will now show that if we can decide whether these problems permit 1-deal negotiation, then we can immediately deduce the answer to the UI problem over $\langle u, Q\rangle$.

First of all, note that if the answer to the UI problem over $\langle u, Q\rangle$ is NO, then both $\mathcal{P}$ and $\overline{\mathcal{P}}$ will permit 1 -deal negotiation. In fact, if it is the case, 0 is the highest value the function $u$ can take, and moving resource $r_{1}$ from an agent to another in $\mathcal{P}$ or $\overline{\mathcal{P}}$ will lead to an optimal allocation if the current allocation is not already optimal. Due to the converse, we can deduce the following: 
- If either $\mathcal{P}$ or $\overline{\mathcal{P}}$ doesn't permit 1-deal negotiation, then the answer to the UI problem is YES

Finally, note that in case where both problems do permit 1-deal negotiation, answering to the UI problem is easy : consider indeed allocation $A=\langle\{\}, \mathcal{R}\rangle$ (resp. $\bar{A}=\langle\mathcal{R},\{\}\rangle$ ). These allocations have a social welfare whose value is $v(A)+v_{0}(A)=\bar{v}(\bar{A})+v_{0}(\bar{A})=0$. Clearly, the answer to the UI problem is YES iff there exists an allocation $A^{\prime}$ such that $v\left(A^{\prime}\right)>0$ or there exists an allocation $\bar{A}^{\prime}$ such that $\bar{v}\left(\bar{A}^{\prime}\right)$. Because of the property of utilities permitting 1-deal negotiation, this is equivalent to finding whether there exists an individually rational 1 -deal $\delta=\left(A, A^{\prime}\right)$ over $\mathcal{P}$ or $\bar{\delta}=\left(\bar{A}, \bar{A}^{\prime}\right)$ over $\overline{\mathcal{P}}$. To summarize:

- If both $\mathcal{P}$ and $\overline{\mathcal{P}}$ do permit 1-deal negotiation then consider allocation $A=\langle\{\}, \mathcal{R}\rangle$ (resp. $\bar{A}=\langle\mathcal{R},\{\}\rangle$ ). If there exists any individually rational 1-deal $\delta=\left(A, A^{\prime}\right)$ over $\mathcal{P}$ or $\bar{\delta}=\left(\bar{A}, \bar{A}^{\prime}\right)$ over $\overline{\mathcal{P}}$, then the answer to the UI problem is YES, otherwise it is NO.

Corollary 1 For any $k \geq 3$ and $n \geq 2$, the problem of checking whether the set $\left\{u_{1}, \ldots, u_{n}\right\}$ of utility functions permits 1-deal negotiation is co-NP-hard if these utilities are represented in k-additive form, posiforms of degree $k$, SLP, or bundle form.

To conclude this section, we could say that for many common expressive representation languages, checking if sets of utilities permit 1-deal negotiation is intractable. Therefore, searching for a condition over sets of utility functions is unrealistic.

As we have shown that no class of utility function was both sufficient and necessary, and as, on top of that, we have just argued that checking conditions on sets of utility functions was intractable in most cases, the best thing we can do is to investigate whether some restricted classes of utility can be identified as being maximal.

\section{Maximal Classes of Utility Functions}

We are now going to prove one of the main results of this paper, namely the surprising fact that the class of modular utility functions is not only sufficient for 1-deal negotiation with money, but also maximal in the sense that no class of utility functions strictly including the modular functions would still be sufficient for 1-deal negotiation. The significance of this result can only be fully appreciated when considered together with the "negative" result on necessary and sufficient conditions discussed in the previous section.

Before stating the main result, we prove the following auxiliary lemma: 
Lemma 2 (Alternative characterisation of modularity) A utility function $u$ is modular iff the following holds for all $R \subseteq \mathcal{R}$ and all $r_{1}, r_{2} \in \mathcal{R}$ with $r_{1}, r_{2} \notin R$ and $r_{1} \neq r_{2}$ :

$$
u\left(R \cup\left\{r_{1}, r_{2}\right\}\right)=u\left(R \cup\left\{r_{1}\right\}\right)+u\left(R \cup\left\{r_{2}\right\}\right)-u(R)
$$

Proof. To show this, let us recall elementary facts about submodular functions. A function $v: \mathcal{R} \rightarrow \mathbb{R}$ is submodular iff $\forall R_{1}, R_{2} \subseteq \mathcal{R}, v\left(R_{1}\right)+v\left(R_{2}\right) \geq v\left(R_{1} \cup R_{2}\right)+v\left(R_{1} \cap R_{2}\right)$. It is also known that $v$ is submodular iff $v\left(R \cup\left\{r_{1}\right\}\right)+v\left(R \cup\left\{r_{2}\right\}\right) \geq v\left(R \cup\left\{r_{1}, r_{2}\right\}\right)-v(R)$ for any $R \subseteq \mathcal{R}, r_{1}, r_{2} \in \mathcal{R} \backslash R$, with $r_{1} \neq r_{2}$ [10, p.662]. Because a function $u$ is modular iff both $u$ and $-u$ are submodular, the lemma holds.

\subsection{Framework With Money}

We are now in a position to prove our theorem on the maximality of the class of modular utility functions with respect to rational negotiation over one resource at a time:

Theorem 5 (Maximality) Let $\mathcal{M}$ be the class of modular utility functions. Then for any class of utility functions $\mathcal{F}$ such that $\mathcal{M} \subset \mathcal{F}, \mathcal{F}$ does not permit 1-deal negotiation.

Proof. First observe that for $|\mathcal{R}| \leq 1$, any utility function is modular, i.e. the theorem holds vacuously in these cases. Therefore, without loss of generality, from now on we assume that there are at least two distinct resources in the system.

The proof is constructive. We will show that for any non-modular utility function $u_{1}$ on $m$ resources, it is possible to construct a modular utility function $u_{2}$ (with $u_{i} \equiv 0$ for all other agents $i$ ) and an initial allocation such that no optimal allocation can be reached by means of 1-deals. This implies that $\mathcal{M} \cup\left\{u_{1}\right\}$ does not permit 1-deals.

Because $u_{1}$ is non-modular, Lemma 2 can be applied in the following way: there exist a bundle $X$ and distinct resources $r_{1}, r_{2} \notin X$ such that $\epsilon$, defined as follows, is not equal to 0 :

$$
\epsilon=u_{1}\left(X \cup\left\{r_{1}\right\}\right)+u_{1}\left(X \cup\left\{r_{2}\right\}\right)-u_{1}(X)-u_{1}\left(X \cup\left\{r_{1}, r_{2}\right\}\right)
$$

From now on, $A_{12 \mid}, A_{\mid 12}, A_{1 \mid 2}$ and $A_{2 \mid 1}$ will refer to allocations in which $r_{1}$ and $r_{2}$ belong to one of the first two agents, and in which resources in $X$ are owned by 1 , and resources in $Y=\mathcal{R} \backslash\left(X \cup\left\{r_{1}, r_{2}\right\}\right)$ by 2 , as shown in the following table.

\begin{tabular}{|c|c|c|}
\hline & Agent 1 & Agent 2 \\
\hline \hline$A_{12 \mid}$ & $\left\{r_{1}, r_{2}\right\} \cup X$ & $Y$ \\
\hline$A_{\mid 12}$ & $X$ & $\left\{r_{1}, r_{2}\right\} \cup Y$ \\
\hline$A_{1 \mid 2}$ & $\left\{r_{1}\right\} \cup X$ & $\left\{r_{2}\right\} \cup Y$ \\
\hline$A_{2 \mid 1}$ & $\left\{r_{2}\right\} \cup X$ & $\left\{r_{1}\right\} \cup Y$ \\
\hline
\end{tabular}




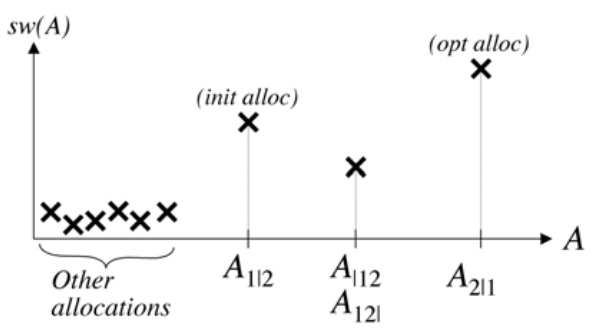

Figure 1: Values of $s w$ for the four allocations (case $\epsilon>0$ ).

Let us build a modular utility function $u_{2}$ defined as follows: $\forall R \in \mathcal{R}$,

$$
u_{2}(R)=\sum_{r \in\left\{r_{1}, r_{2}\right\} \cap R} \alpha_{r}+\sum_{r \in R \cap Y} \omega-\sum_{r \in R \cap X} \omega
$$

with $\omega=14 \times \max \left|u_{1}\right|+1$. Let $\Omega=u_{2}(Y)=|Y| \times \omega$. As the rest of the proof shall reveal, the value of $\omega$ has been chosen such that the social welfare of each of these four allocations is greater than that of any other allocation. Of course, this will imply that the optimal allocation has to be among these four. The values of $\alpha_{r_{1}}$ and $\alpha_{r_{2}}$ will be chosen later. The social welfare of each of these four allocations can then be written as follows:

$$
\begin{aligned}
& s w\left(A_{\mid 12}\right)=\Omega+\alpha_{r_{1}}+\alpha_{r_{2}}+u_{1}(X) \\
& s w\left(A_{12 \mid}\right)=\Omega+u_{1}\left(X \cup\left\{r_{1}, r_{2}\right\}\right) \\
& s w\left(A_{1 \mid 2}\right)=\Omega+\alpha_{r_{2}}+u_{1}\left(X \cup\left\{r_{1}\right\}\right) \\
& s w\left(A_{2 \mid 1}\right)=\Omega+\alpha_{r_{1}}+u_{1}\left(X \cup\left\{r_{2}\right\}\right)
\end{aligned}
$$

It remains to be shown that depending on the value of $\epsilon$, we can always choose an initial allocation among these four and values of $\alpha_{r_{1}}$ and $\alpha_{r_{2}}$ such that (1) this initial allocation does not have optimal social welfare, (2) there is only one rational deal from this allocation, (3) this deal leads to the optimal allocation but however (4) this rational deal would involve more than one resource. We will have to consider two cases for equation (4): the case of $\epsilon>0$ and the case of $\epsilon<0$.

(1st case) Suppose $\epsilon>0$. Let us choose $\alpha_{r_{1}}=u_{1}\left(X \cup\left\{r_{1}\right\}\right)-u_{1}(X)-\frac{\epsilon}{4}$ and $\alpha_{r_{2}}=$ $u_{1}\left(X \cup\left\{r_{1}, r_{2}\right\}\right)-u_{1}\left(X \cup\left\{r_{1}\right\}\right)+\frac{\epsilon}{4}$.

Let us first show that the four allocations have a greater social welfare than any other. With the help of equation (4), observe that both $\left|\alpha_{r_{1}}\right|$ and $\left|\alpha_{r_{2}}\right|$ are less than $3 \times \max \left|u_{1}\right|$. Thus, all four allocations have a social welfare of at least $\Omega-\left|\alpha_{r_{1}}\right|-\left|\alpha_{r_{2}}\right|-\max \left|u_{1}\right| \geq$ $\Omega-7 \times \max \left|u_{1}\right|>\Omega-\frac{\omega}{2}$. All other allocations have a social welfare lower than 


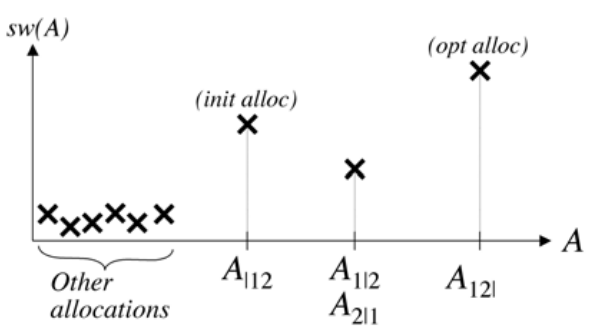

Figure 2: Values of $s w$ for the four allocations (case $\epsilon<0$ ).

$\Omega-\omega+\left|\alpha_{r_{1}}\right|+\left|\alpha_{r_{2}}\right|+\max \left|u_{1}\right| \leq \Omega-\omega+7 \times \max \left|u_{1}\right|<\Omega-\frac{\omega}{2}$. Thus, the social welfare of each of the four allocations is greater than that of any other allocation.

Now let us show that $A_{2 \mid 1}$ is the optimal allocation, as illustrated in Figure 1. More precisely, let us show that $s w\left(A_{\mid 12}\right)<s w\left(A_{1 \mid 2}\right)$, that $s w\left(A_{12 \mid}\right)<s w\left(A_{1 \mid 2}\right)$ and that $s w\left(A_{1 \mid 2}\right)<s w\left(A_{2 \mid 1}\right)$. By substituting the values of $\alpha_{r_{1}}$ and $\alpha_{r_{2}}$ and using equation (4), the social welfare of each allocation can be written as follows:

$$
\begin{aligned}
s w\left(A_{\mid 12}\right)= & \Omega+u_{1}\left(X \cup\left\{r_{1}, r_{2}\right\}\right) \\
s w\left(A_{12 \mid}\right)= & \Omega+u_{1}\left(X \cup\left\{r_{1}, r_{2}\right\}\right) \\
s w\left(A_{1 \mid 2}\right)= & \Omega+u_{1}\left(X \cup\left\{r_{1}, r_{2}\right\}\right)+\frac{\epsilon}{4} \\
s w\left(A_{2 \mid 1}\right)= & \Omega+u_{1}\left(X \cup\left\{r_{1}\right\}\right)+u_{1}\left(X \cup\left\{r_{2}\right\}\right) \\
& -u_{1}(X)-\frac{\epsilon}{4} \\
= & \Omega+u_{1}\left(X \cup\left\{r_{1}, r_{2}\right\}\right)+\frac{3}{4} \epsilon
\end{aligned}
$$

Here, $A_{2 \mid 1}$ is clearly the optimal allocation. If we choose $A_{1 \mid 2}$ as the initial allocation, then the only 1-deals involving resources $r_{1}$ or $r_{2}$ are $\delta\left(A_{1 \mid 2}, A_{12 \mid}\right)$ and $\delta\left(A_{1 \mid 2}, A_{\mid 12}\right)$. These deals decrease social welfare, and thus are not individually rational by Lemma 1 . Thus, it is not possible to reach the optimal allocation $A_{2 \mid 1}$ starting from $A_{1 \mid 2}$ using only 1-deals.

(2nd case) Suppose $\epsilon<0$. Let us choose $\alpha_{1}=u_{1}\left(X \cup\left\{r_{1}\right\}\right)-u_{1}(X)-\frac{\epsilon}{4}$ and $\alpha_{2}=$ $u_{1}\left(X \cup\left\{r_{2}\right\}\right)-u_{1}(X)-\frac{\epsilon}{4}$.

Note that again, both $\left|\alpha_{r_{1}}\right|$ and $\left|\alpha_{r_{2}}\right|$ are less than $3 \times \max \left|u_{1}\right|$. Thus, by the same argument as in the first case, the four allocations all have greater social welfare than any other allocation. 
The optimal allocation is now $A_{12 \mid}$. To see this, let us show that $s w\left(A_{1 \mid 2}\right)<s w\left(A_{\mid 12}\right)$, that $s w\left(A_{2 \mid 1}\right)<s w\left(A_{\mid 12}\right)$, and that $s w\left(A_{\mid 12}\right)<s w\left(A_{12 \mid}\right)$ as illustrated in Figure 2.

$$
\begin{aligned}
s w\left(A_{\mid 12}\right)= & \Omega+u_{1}\left(X \cup\left\{r_{1}\right\}\right)+u_{1}\left(X \cup\left\{r_{2}\right\}\right) \\
& -u_{1}(X)-\frac{\epsilon}{2} \\
s w\left(A_{12 \mid}\right)= & \Omega+u_{1}\left(X \cup\left\{r_{1}, r_{2}\right\}\right) \\
= & \Omega+u_{1}\left(X \cup\left\{r_{1}\right\}\right)+u_{1}\left(X \cup\left\{r_{2}\right\}\right) \\
& -u_{1}(X)-\epsilon \\
s w\left(A_{1 \mid 2}\right)= & \Omega+u_{1}\left(X \cup\left\{r_{1}\right\}\right)+u_{1}\left(X \cup\left\{r_{2}\right\}\right) \\
& -u_{1}(X)-\frac{\epsilon}{4} \\
s w\left(A_{2 \mid 1}\right)= & \Omega+u_{1}\left(X \cup\left\{r_{1}\right\}\right)+u_{1}\left(X \cup\left\{r_{2}\right\}\right) \\
& -u_{1}(X)-\frac{\epsilon}{4}
\end{aligned}
$$

Here, $A_{12 \mid}$ is clearly the optimal allocation. If we choose $A_{\mid 12}$ as the initial allocation, then the only 1-deals involving $r_{1}$ or $r_{2}$ are $\delta\left(A_{\mid 12}, A_{1 \mid 2}\right)$ and $\delta\left(A_{\mid 12}, A_{2 \mid 1}\right)$. These deals decrease social welfare, and thus are not individually rational by Lemma 1 . Thus, it is not possible to reach the optimal allocation $A_{12 \mid}$ starting from $A_{\mid 12}$ using only 1-deals.

Why is this result significant? As argued earlier, while the full abstract negotiation framework introduced at the beginning of this paper would be difficult to implement, designing a system that only allows for pairs of agents to agree on deals over one resource at a time is entirely feasible. As we would like to be able to guarantee socially optimal outcomes in as many cases as possible, also for such a restricted negotiation system, we would like to be able to identify the largest possible class of utility functions for which such a guarantee can be given. However, our discussion in Section 6.2 has shown that there can be no class of utility functions that exactly characterises the class of negotiation problems for which negotiating socially optimal allocations by means of rational 1-deals is always possible. Still, there are classes of utility functions that permit 1-deal negotiation. As shown by Theorem 2, the class of modular functions is such a class and it is a very natural class to consider. An obvious question to ask is therefore whether this class can be enlarged in any way without losing the desired convergence property.

Our proof of Theorem 5 settles this question by giving a negative answer: For any agent with a non-modular utility function there exist modular utility functions (for the other agents) and an initial allocation such that rational 1-deals alone do not suffice to negotiate an allocation of resources with maximal social welfare. There may well be further such classes (that are both sufficient and maximal), but the class of modular functions is one that is particularly natural and useful in the context of modelling agent preferences. 


\subsection{Framework Without Money}

Can the result of the framework with money be translated in our framework without money? We are now going to show that this is the case: any class of modular utility functions with $\alpha, \beta$ shared values is not only sufficient for 1-deal negotiation, but also maximal in the sense that no class of utility functions strictly including that class would still be sufficient for 1-deal negotiation.

Theorem 6 (Maximality) Let $\mathcal{M}_{\alpha, \beta}$ be a class of modular functions with shared $\alpha, \beta$ values. Then for any class of utility functions $\mathcal{F}$ such that $\mathcal{M}_{\alpha, \beta} \subset \mathcal{F}, \mathcal{F}$ does not permit 1-deal negotiation.

Proof. The proof is constructive, and involves two agents. We will show that for any function $u_{1}$ not belonging to the class of modular functions with shared $\alpha, \beta$-values, it is possible to construct a modular utility function $u_{2}$ and to choose an initial allocation such that the optimal allocation cannot be reached by means of cooperative rational 1-deals. This will imply that $\mathcal{M}_{\alpha, \beta} \cup\left\{u_{1}\right\}$ does not permit 1-deal negotiation.

First note that for any modular function with shared $\alpha, \beta$-values, iff $\forall X \subset \mathcal{R}, \forall r \in$ $\mathcal{R} \backslash X: u(X \cup\{r\})=u(X)+v$, with $v \in\left\{\alpha_{r}, 0, \beta_{r}\right\}$. Let $u_{1}$ be an arbitrary function, not belonging to the class $\mathcal{M}_{\alpha, \beta}$, that is, there exists $X \subset \mathcal{R}$ and a resource $r_{1} \in \mathcal{R} \backslash X$ such that $u\left(X \cup\left\{r_{1}\right\}\right)=u(X)+v$, with $v \notin\left\{\alpha_{r_{1}}, 0, \beta_{r_{1}}\right\}$. (Recall that $\alpha_{r_{1}}>0$ and $\beta_{r_{1}}<0$ ). Now, let $u_{2} \in \mathcal{M}_{\alpha, \beta}$ defined as follows: $\forall R \in \mathcal{R}$ :

$$
u_{2}(R)=u_{2}(\{\})+\sum_{r \in R \cap X} \beta_{r}+\sum_{r \in R \cap \mathcal{R} \backslash\left(X \cup\left\{r_{1}\right\}\right)} \alpha_{r}+\sum_{r \in R \cap\left\{r_{1}\right\}} z
$$

with $z$ to be chosen in $\left\{\alpha_{r_{1}}, \beta_{r_{1}}\right\}$ We now consider the two allocations where agent 1 owns all resources in $X$, and agent 2 owns all resources in $\mathcal{R} \backslash\left(X \cup\left\{r_{1}\right\}\right)$. Amongst these allocations, let $A_{r_{1} \mid}$ (resp. $A_{\mid r_{1}}$ ) be the one where agent 1 (resp. agent 2) owns in addition resource $r_{1}$. It remains to be shown that no cooperative 1-deal is possible. This can be done by considering the different cases. We start by observing that agent 1 cannot pass a single resource $r \in X$ to agent 2 (because $u_{2}$ would decrease of $\left|\beta_{r}\right|$ ), and that agent 2 cannot pass a single resource $r \in \mathcal{R} \backslash\left(X \cup\left\{r_{1}\right\}\right)$ because in this case $u_{2}$ would decrease of the value $\alpha_{r}$. Let us now consider the case of 1-deals involving resource $r_{1}$. Note that $s w\left(A_{r_{1} \mid}\right)-s w\left(A_{\mid r_{1}}\right)=v-z$. There are now four different cases to consider depending on the value of $v$.

(case 1): $v>\alpha_{r_{1}}$. By choosing $z=\alpha_{r_{1}}$, we prevent agent 2 from passing $r_{1}$ on to agent 1 (because $u_{2}$ would decrease of $\left.\alpha_{r_{1}}\right)$, but still we have $s w\left(A_{r_{1} \mid}\right)>s w\left(A_{\mid r_{1}}\right)$ (because $v-z>0)$.

(case 2): $v<\beta_{r_{1}}$. By choosing $z=\beta_{r_{1}}$, we prevent agent 1 from passing $r_{1}$ on to agent 2 (because $u_{1}$ would decrease of $\left|\beta_{r_{1}}\right|$ ), but still we have $s w\left(A_{r_{1} \mid}\right)<s w\left(A_{\mid r_{1}}\right)$ (because 
$v-z<0)$.

(case 3): $0<v<\alpha_{r_{1}}$. By choosing $z=\alpha_{r_{1}}$, we have $s w\left(A_{r_{1} \mid}\right)<s w\left(A_{\mid r_{1}}\right)$ (because $v-z<0$ ), but still agent 1 cannot give $r_{1}$ to agent 2 (because $u_{1}$ would decrease of $v$ ). (case 4): $\beta_{r_{1}}<v<0$. By choosing $z=\beta_{r_{1}}$, we have $s w\left(A_{r_{1} \mid}\right)>\operatorname{sw}\left(A_{\mid r_{1}}\right)$ (because $v-z>0$ ), but still agent 2 cannot give $r_{1}$ to agent 1 (because $u_{2}$ would decrease of $|v|$ ).

\section{Conclusion}

This paper makes a contribution to the theoretical analysis of a negotiation framework where rational but myopic agents agree on a sequence of deals regarding the reallocation of a number of discrete resources. We have shown that the use of modular utility functions to model agent preferences is a sufficient condition, if side-payments are allowed, to guarantee final allocations with maximal social welfare in case agents only negotiate 1-deals (involving one resource each). When no side-payments are permitted, any class of $\mathcal{M}_{\alpha, \beta}$ functions would be sufficient. We have then seen that this is, however, not a necessary condition for optimal outcomes (being it with or without money) and, indeed, there can be no condition on (individual) utility functions that would be both necessary and sufficient in this sense. Furthermore, we have shown that, while a necessary and sufficient condition on sets of utility functions obviously does exist, checking it is intractable for most representations commonly used in multiagent resource allocation settings. We have therefore concentrated on showing that the class of modular (resp. with shared $\alpha, \beta$ values) functions is maximal in the framework with money (resp. without money), i.e. no strictly larger class of functions would still permit an optimal allocation to be found by means of rational 1-deals in all cases.

We consider this not only a surprising result, but also a useful characterisation of negotiation domains that can be handled reliably using simple negotiation protocols, catering only for Contract Net-like deals over single items between pairs of agents, rather than the full range of multilateral deals forseen in the abstract framework. Such theoretical results affect both the design of agents and of negotiation mechanisms. For instance, if a given mechanism can only handle 1-deals, then it may be inappropriate to design myopic agents with very rich preference structures to use such a mechanism.

In a companion paper [3], we prove a generalisation of Theorem 1 which shows that rational deals involving at most $k$ resources each are sufficient for convergence to an optimal allocation in case all utility functions are additively separable with respect to a common partition of $\mathcal{R}$ (i.e. synergies across different parts of the partition are not possible and overall utility is defined as the sum of utilities for the different sets in the partition [8]), and each set in this partition has at most $k$ elements. The arguments against the existence of sufficient conditions for negotiation over $k$ items at a time that are also necessary gen- 
eralise in the expected manner. An important issue that remains to be investigated in the future therefore is to see whether it is possible to derive a similar maximality property as the one proved in this paper for this richer class of utility functions.

\section{References}

[1] K. J. Arrow, A. K. Sen, and K. Suzumura, editors. Handbook of Social Choice and Welfare, volume 1. North-Holland, 2002.

[2] Endre Boros and Peter L. Hammer. Pseudo-boolean optimization. Discrete Applied Mathematics, 123(1-3):155-225, 2002.

[3] Y. Chevaleyre, U. Endriss, J. Lang, and N. Maudet. Negotiating over small bundles of resources. In Proc. AAMAS-2005. ACM Press, 2005.

[4] Yann Chevaleyre, Ulle Endriss, Sylvia Estivie, and Nicolas Maudet. Multiagent resource allocation with $k$-additive utility functions. In Proc. of the DIMACSLAMSADE Workshop on Computer Science and Decision Theory, 2004.

[5] P. Cramton, Y. Shoham, and R. Steinberg, editors. Combinatorial Auctions. MIT Press, 2005. To appear.

[6] P. E. Dunne, M. Wooldridge, and M. Laurence. The complexity of contract negotiation. Artificial Intelligence, 2005. To appear.

[7] U. Endriss, N. Maudet, F. Sadri, and F. Toni. On optimal outcomes of negotiations over resources. In Proc. AAMAS-2003. ACM Press, 2003.

[8] P. C. Fishburn. Utility Theory for Decision Making. John Wiley and Sons, 1970.

[9] M. Grabisch. $k$-order additive discrete fuzzy measures and their representation. Fuzzy Sets and Systems, 92:167-189, 1997.

[10] G. L. Nemhauser and L.A. Wolsey. Integer and Combinatorial Optimization. John Wiley and Sons, 1988.

[11] J. S. Rosenschein and G. Zlotkin. Rules of Encounter. MIT Press, 1994.

[12] T. W. Sandholm. Contract types for satisficing task allocation: I Theoretical results. In Proc. AAAI Spring Symposium: Satisficing Models, 1998.

[13] R. G. Smith. The contract net protocol: High-level communication and control in a distributed problem solver. IEEE Transactions on Computers, C-29(12):1104-1113, 1980 . 
Restricted Classes of Utility Functions for Simple Negotiation Schemes [...]

[14] M. Wooldridge. An Introduction to MultiAgent Systems. John Wiley and Sons, 2002. 